\title{
Warum wir eine Biotechnologie-Agenda brauchen
}

DOI: $10.1007 / \mathrm{s} 12268-018-0979-5$

(C) Springer-Verlag 2018

Nächstes Jahr feiert der Begriff Biotechnologie einen runden Geburtstag. Mit wandelnder Bedeutung wird er dann 100 Jahre genutzt. Viele Tausende Jahre länger werden biotechnologische Prozesse natürlich vom Menschen schon eingesetzt, um Nahrungsmittel zu veredeln und Prozesse zu beschleunigen. Der Nobelpreis für Chemie 2018 würdigte die gerichtete Veränderung von Enzymen zum optimalen Einsatz in zahlreichen Anwendungen, wie z. B. in Wasch- und Lebensmitteln. Ein Prozess, der durch die neuen Methoden der Genomeditierung noch erheblich beschleunigt werden kann. Auch im Bereich Gesundheit eröffnen biotechnologische Arzneimittel heutzutage ganz neue Therapieoptionen.

Warum ist die Biotechnologie also nicht ein „Rockstar" der Technik und in aller Munde? Bundeswirtschaftsminister Peter Altmaier erkennt das Potenzial der Biotechnologie und sagte auf dem Forschungsgipfel 2017: „Ich frage mich, ob wir nach der Digitalen Agenda nicht auch eine Biotechnologie-Agenda brauchen“. Auch das Bundesforschungsministerium hat sich für eine solche Agenda engagiert, die aber nicht so heißen sollte. Im Koalitionsvertrag wurde festgehalten, eine Agenda mit dem etwas sperrigen Namen „Von der Biologie zur Innovation“ ins Leben zu rufen, die auch in der neuen Hightech-Strategie einen Platz gefunden hat. Vielleicht ließe sich den Errungenschaften und dem Potenzial der Biotechnologie besser Rechnung tragen, wenn die Agenda auch so heißen würde.

Um den Inhalt der Agenda mitzugestalten, haben wir bei BIO Deutschland schon frühzeitig Maßnahmenpakete in einer Handreichung zusammengefasst [1].

Ein wichtiger Punkt ist das Agenda-Setting. Die Themen Biotechnologie und Biologisierung sollten auf die Agenda des öffentlichen Diskurses und der politischen Debatten gesetzt werden. Um das zu erreichen, müssen wir den Begriff Biotechnologie auch nut- zen. In einer Pressemitteilung vom 2. Oktober 2018 [2] zur Ankündigung der nächsten Bioökonomie-Strategie aus dem Bundeslandwirtschaftsministerium ist das Wort Biotechnologie bzw. biotechnologisch kein einziges Mal zu lesen. Es geht stattdessen um „umweltschonende Produktionsverfahren“ oder "die Entwicklung innovativer biobasierter Alternativen zu bestehenden Produkten und Prozessen“. Vonseiten der Nichtregierungsorganisationen ist zwar häufig die Kritik zu hören, dass die Industrie zu stark auf Technologie zur Lösung gesellschaftlicher Herausforderungen fokussiert. Es muss dennoch klar sein, dass technologische Ansätze immer Teil der Lösung sein werden, sei es beim Klima- und Umweltschutz oder bei der Gesundheit.

Auch müssen wir unsere Standortkompetenz im Bereich Biotechnologie erhalten und ausbauen, z. B. durch maßgeschneiderte (Aus-)Bildungsangebote für moderne Bioingenieure, Biochemiker und Biotechnologen sowie die entsprechenden Fachkräfte im Labor, einschließlich der Fähigkeiten zur Verarbeitung von Big Data und lernender Systeme nach biologischem Vorbild.

Die auf Erdöl basierende Wirtschaft ist über Jahrzehnte optimiert worden. Viele Kosten werden externalisiert und schlagen deshalb im Vergleich nicht zu Buche. Es ist nicht zu erwarten, dass neue, nachhaltige und $\mathrm{CO}_{2}-$ neutrale biotechnologische Prozesse von Anfang an auf Kostenebene konkurrenzfähig sind. Daher müssen zeitlich begrenzte ordnungspolitische Maßnahmen erarbeitet werden, die Investitionsanreize sowie eine gewisse Investitionssicherheit schaffen, um die Einführung und Etablierung neuer biotechnologischer Verfahren zu ermöglichen.

Kooperation ist wichtig. Als Schlüsseltechnologie stellt die Biotechnologie für viele Traditionssektoren eine neue Kundengruppe dar. Die Biotechnologie-Industrie mit ihren Produktionsstraßen, Fermentationsanlagen, Mikrosensoren, mit ihren automatisierten und miniaturisierten oder kybernetischen Prozessen ist geeignet, abgewanderte, schrumpfende oder sich wandelnde Märkte zu beleben. Daher ist die Förderung von Allianzen und neuen Geschäftsmodellen wichtig.

Innovative Gründerinnen und Gründer sowie Innovatoren in kleinen und mittelständischen Unternehmen sind der Grundstein für eine standortgebundene Wertschöpfung in Deutschland. Daher brauchen wir den Aufbau eines ausgeglichenen Venture-Capital-Umfelds und die Mobilisierung privaten Kapitals, um den Finanzierungsbedarf von innovativen Geschäftsmodellen bis zum Markteintritt zu decken.

Dieser kurze Abriss skizziert die aus unserer Sicht notwendigen Maßnahmen, die in der Agenda „Von der Biologie zur Innovation“ gebündelt werden müssen, um unser Ziel zu erreichen: nämlich der erfolgreiche Einsatz der Biotechnologie für Klima- und Umweltschutz, nachhaltige Produktionsprozesse, Produkte und Mobilität sowie für ausreichend nährstoffreiche Lebensmittel und globale Gesundheit.

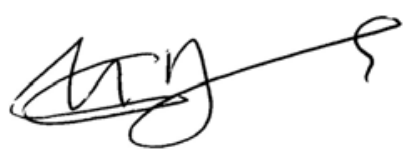

Viola Bronsema, Geschäftsführerin BIO Deutschland e. V.

Korrespondenzadresse:

Dr. Viola Bronsema

Biotechnologie-Industrie-Organisation

Deutschland e. V. (BIO Deutschland e. V.)

Am Weidendamm 1a

D-10117 Berlin

Tel.: 030-72625-130

bronsema@biodeutschland.org

\section{Literatur}

[1] Handreichung für eine ressortübergreifende Agenda „Von der Biologie zur Innovation“ (Biotechnologie-Agenda), www.biodeutschland.org/de/positionspapiere/handreichungfuer-eine-ressortuebergreifende-agenda-von-der-biologie-zurinnovation-biotechnologie-agenda.html 